\section{PABS: Patienten-Anlauf- und -Beratungsstelle der Medizinischen Gesellschaft Basel}

W. Tschan

Die MedGes (Medizinische Gesellschaft Basel) hat mit Beschluss der Mitgliederversammlung vom 29. März 2001 den Aufbau der PABS in die Wege geleitet [1]. Der Autor versucht mit diesem Artikel, die Hintergründe und Überlegungen aufzuzeigen, die zur Schaffung dieser Instanz geführt haben. Weiter soll die vorgesehene Arbeitsweise dieser Beratungsstelle dargelegt werden. Die mit der Arbeit der PABS verbundenen Konfliktbereiche sollen reflektiert und der "professional community" zur Kenntnis gebracht werden. Es werden mögliche Implikationen für die Standesordnung FMH diskutiert. In einem Ausblick werden weitere Schritte und Massnahmen aufgezeigt, welche sich aus dieser Diskussion ergeben. Die Arbeiten der Medizinischen Gesellschaft Basel stehen nicht isoliert da. Im Kanton Schaffhausen sind ähnliche Bestrebungen im Gange. In vielen Bereichen ausserhalb des Gesundheitswesens wurden in den zurückliegenden Jahren spezialisierte Beratungsstellen gegen sexuelle Gewalt und Machtmissbräuche eingerichtet (vgl. beispielsweise Universität Basel, Evangelische Landeskirche Bern und Jura, Musikausbildung, Sport und Freizeit). Zudem hat die Schweizerische Gesellschaft für Psychiatrie und Psychotherapie in einem kürzlich verabschiedeten Grundsatzpapier analoge Massnahmen empfohlen. Das Bundesamt für Gesundheitswesen setzt sich im Rahmen von Qualitätssicherungsmassnahmen ebenfalls mit diesen Aspekten auseinander. Eine vorausschauende Sichtweise verhilft der Ärzteschaft zu einer zielgerichteten Vorgehensweise. Der Autor war sowohl in der vorbereitenden Arbeitsgruppe mit der Konzeption involviert als nun auch als Mitglied der Steuerungsgruppe (SG-PABS) mit deren Umsetzung beauftragt.

Korrespondenz:

Dr. med. Werner Tschan

Neuensteinerstrasse 7

CH-4053 Basel

E-mail: tschankast@bluewin.ch
PABS-Hotline

0612799149

ab sofort operationell

einsatzbereit

\section{Vision}

Dass Fachleuten in Ausübung ihres Berufes Fehler unterlaufen, ist nichts Neues. Ärzte sind davon nicht ausgenommen. Der hippokratische Eid - immerhin 2400 Jahre alt - mag belegen, dass insbesondere die Thematik der sexuellen Missbräuche die Ärzteschaft nicht erst jetzt beschäftigt. Dennoch haben sich in den zurückliegenden Jahren viele Schwierigkeiten im Zusammenhang mit der Thematik ergeben. Bei den zu diskutierenden Massnahmen geht es nicht um Disziplinierung und Bestrafung von Fehlbaren, sondern es geht in erster Linie darum, dass entsprechend dem Ethos unserer ärztlichen Disziplin notwendige Hilfestellungen angeboten werden sollen. Der Vorstand der MedGes erkannte im Anschluss an ein Konklave die Notwendigkeit einer spezialisierten Beratungsstelle in Zusammenhang mit sexuellen Missbräuchen durch Kolleginnen und Kollegen. Die bisherigen Strukturen, sowohl die Ombudsstelle als auch die Standesverfahren vor Ehrenrat, erwiesen sich als ungeeignet, die anstehenden Probleme angesichts der oft komplexen Fragestellungen zu lösen. Insbesondere war sich der Vorstand MedGes der Verantwortung bewusst, welche wir als Berufsorganisation gegenüber betroffenen Opfern und ihren Angehörigen haben, wenn es zu sexuellen Missbräuchen durch ärztliche Kolleginnen und Kollegen kommt.

Vom Vorstand wurde eine 7köpfige Arbeitsgruppe bestellt, welche den Basler Kantonsarzt Dr. med. Bruno Bucheli zur Mitarbeit gewinnen konnte. In einem ersten Schritt wurde der Ist-Zustand analysiert und ein möglicher Soll-Zustand formuliert, welcher sich am «best possible standard» orientierte. Dass damit für viele Kolleginnen und Kollegen Neuland betreten wird, ist verständlich. In Absprache mit dem Vorstand wurde für die Beratungsstelle eine Vision formuliert, welche einen Denkprozess einleiten soll, der dann schliesslich zu einem Konsens über die zu ergreifenden Massnahmen führen kann. Diese Vorgehensweise scheint auf breite Akzeptanz gestossen zu sein und hat sich als befruchtend erwiesen. Gleichzeitig hat die Arbeitsgruppe von Beginn an klargemacht, dass nicht eine auf alle Zeiten gültige Lösung präsentiert werden kann, sondern dass ein pragmatischer und praktikabler Weg angestrebt wird, der gegebenenfalls aufgrund von Feedbacks zu überdenken sein wird. Es soll auch nicht vergessen werden, dass die bis heute gewachsenen Strukturen der MedGes auf ihre Gründungszeit von 1860 zurückgehen - so wie diejenigen der FMH auch bereits ihr hundertjähriges Bestehen feiern dürfen. Das Basler Gesetz betreffend Ausübung der Berufe der Medizinalpersonen stammt vom 26. Mai 1879 [2]. Bereits in den Anfängen unserer Berufsverbände suchten unsere Kollegen nach Wegen, 


\section{Richtlinien für die PABS bei sexuellen Missbräuchen durch Ärztinnen und Ärzte}

Sexuelle Handlungen mit Patienten oder Patientinnen stellen eine schwere Verletzung jeder ärztlichen Tätigkeit dar. Es werden damit grundlegende Bedingungen einer fachlichen Tätigkeit in einer Art und Weise missachtet, dass die weitere Berufstätigkeit in Frage gestellt ist.

Betroffene und ihre Angehörigen können sich an die PABS (Patienten-Anlauf- und -Beratungsstelle) der Medizinischen Gesellschaft Basel wenden, welche in solchen Fällen eine Beratung übernimmt. Betroffene können zu allen Gesprächen bei der PABS durch eine Vertrauensperson begleitet werden.

- Sexuelle Belästigungen und Missbräuche sind diskriminierend und verletzen die Würde und Rechte von Patientinnen und Patienten. Versuche zur Erlangung einer sexuellen Handlung gelten in Analogie als Übergriffe. Sexuelle Handlungen gelten als Kunstfehler jeder ärztlichen Tätigkeit.

- Wenn eine Person eine ärztliche Behandlung sucht, wird ein Auftragsverhältnis begründet. In aller Regel beginnt die fachliche Beziehung bereits mit der Vereinbarung eines Konsultationstermines.

- Die Fachperson ist für die Einhaltung der fachlichen Grenzen verantwortlich. Dies kann nie Aufgabe und Sache von Patientinnen oder Patienten sein. Es ist daher unerheblich, von wem die Initiative zur Aufnahme eines sexuellen Kontaktes ausgeht.

- Das Vertrauensverhältnis zwischen Arzt und Patient wirkt auch über das eigentliche Behandlungsende hinaus fort. Die Karenzfrist ist abhängig von der Zeitdauer, der Intensität und der Art der Behandlung und sollte nicht unter 2 Jahren betragen.

- Sexuelle Missbräuche können in unterschiedlicher Form auftreten:

- anzügliche und peinliche Bemerkungen;

- zweideutige Aufforderungen;

- wiederholte unerwünschte Einladungen;

- sexuell gefärbte Bemerkungen über körperliche Vorzüge oder Schwächen;

- sexistische Sprüche und Witze, die Personen aufgrund ihres Geschlechtes herabwürdigen;

- aufdringliche Blicke, Beobachten von Patientinnen oder Patienten, während sie sich umziehen;

- Vorzeigen von Bildern pornographischen Inhaltes;

- zudringliche Körperkontakte, erotische Berührungen und Umarmungen;

- Annäherungsversuche;

- sexuelle Handlungen.
Bestätigen sich die Vorwürfe, müssen Betroffene entscheiden können, welche weiteren Schritte sie einzuleiten gedenken. Die Beratung durch die PABS soll dabei diejenigen Schritte unterstützen, die für die betreffende Person in der jeweiligen Situation optimal sind. Betroffene sollen zu keinen Schritten gedrängt werden, für die sie noch nicht bereit sind.

Mögliche Schritte können dabei sein:

- nichts tun;

- Aufnehmen einer Behandlung, allfällige Schritte werden allenfalls später eingeleitet;

- Kontaktaufnahme mit dem Täter, Konfrontationsgespräche;

- aussergerichtlicher Vergleich;

- Klage an den Ehrenrat der Medizinischen Gesellschaft Basel;

- Meldung an das Sanitätsdepartement BaselStadt als Aufsichtsorgan;

- Kompensationen aus dem OHG (Opferhilfegesetz);

- Einleitung zivilrechtlicher Schritte, insbesondere wegen Verletzung von OR 394 ff;

- Einleitung strafrechtlicher Schritte, insbesondere wegen Verletzung von Art. 193 StGB.

Die einzelnen Schritte können beliebig kombiniert werden.

Ratsuchende sollen durch die PABS darüber aufgeklärt werden, dass die Informationen und Angaben innerhalb der PABS unter den Mitarbeitern ausgetauscht werden. In jedem Falle sollen Betroffene einen kurzen persönlichen Bericht über das Vorgefallene erstellen, auch wenn sie zum Zeitpunkt der Beratung durch die PABS keine weiteren Schritte zu unternehmen gedenken. Ohne ausdrückliche Autorisation durch Betroffene verbleiben diese Schriftstücke bei der PABS. Sie werden durch die Medizinische Gesellschaft so archiviert, dass ein unbefugter Zugang ausgeschlossen ist.

Die Weitergabe von Informationen und Unterlagen an den Ehrenrat oder an andere Stellen erfolgt nur nach ausdrücklicher Autorisation durch die betroffene Person. 
die Gefahr von sexuellen Missbräuchen zu bannen. Die PABS wird ihr Augenmerk jedoch nicht nur auf die ärztlichen Kolleginnen und Kollegen zu richten haben, sondern auch diejenigen Fachleute und Institutionen miteinschliessen, denen wir unsere Patienten zur Behandlung zuweisen. Dazu gehören Spitexdienste genau so wie Physiotherapien, ferner die öffentlichen und privaten Spitäler wie auch die Institutionen zur Betreuung von Betagten und Behinderten.

\section{Qualitätssicherung}

Ohne Wahrnehmung der Zahl von betroffenen Opfer wird zwangsläufig auch kein Handlungsbedarf erkannt. Die PABS wurde aus der Opferperspektive konzipiert. Die «victimisation» erfolgt jeweils im Rahmen einer ärztlichen Behandlung, allenfalls auch nach einer ärztlichen Überweisung. Die etwas schwerfällige deutsche Übersetzung "Opferwerdung" beschreibt den Prozess, wenn ein Mensch durch sexuelle Handlungen seelisch verletzt wird [3]. Die pathogenetischen Mechanismen sind durch die Psychotraumatologieforschung hinlänglich belegt $[4,5]$. Die diagnostischen Kriterien für eine posttraumatische Belastungsstörung (PTSD) treffen dabei nur für einen Teil der Opfer zu, für andere nicht. Es wurde durch die Verfasser des PTSD-Konzeptes erkannt, dass zusätzliche diagnostische Entitäten notwendig sind, um diese Störungsbilder korrekt zu erfassen [6]. Die primäre Viktimisierung resultiert aus der unmittelbaren Traumatisierung. Die Folgen können zu lebenslang nachwirkenden Symptomen führen, insbesondere weil der Täter als Vertrauensperson handelte. Die sekundäre Viktimisierung erfolgt durch unsachgemässes, unsensibles und zusätzlich belastendes Verhalten durch beurteilende Fachleute, Behörden oder Angehörige. Als tertiäre Viktimisierung werden die Langzeitfolgen verstanden, die sich durch die Fixierung in eine Opferrolle ergeben, wo betreffende Personen das Gefühl entwickeln, keine Chance mehr zu haben, je wieder aus diesem Teufelskreis des «Opferseins» herausfinden zu können.

Aus unterschiedlichen Gründen wird der Begriff "Opfer" von vielen als problematisch empfunden. Sie befürchten, damit in eine passive, als Unfähigkeit oder Schwäche deklarierte Position hineingestellt zu werden. Die PABS verzichtete deshalb auf die Verwendung dieses Begriffes, um allfällige Stigmatisierungen $\mathrm{zu}$ vermeiden. Ebensowenig ist von Opferunterstützung oder -hilfe die Rede, obwohl faktisch die PABS dem entspricht.

Für unser Land existieren keinerlei epidemiologische Daten, welche die Inzidenz von sexuellen Missbräuchen durch Fachleute belegen. Ist man jedoch in der Opferberatung tätig - in der Schweiz existieren immerhin über 750 Beratungsstellen für sexuelle Gewalt [7], allerdings nicht spezialisiert auf fachliche Missbräuche -, macht man rasch die Evidenzerfahrung, dass die bisherige Handhabung im Kontext von fachlichen Übergriffen völlig unzureichend ist. Betroffene beklagen sich über vielerlei Unzulänglich- keiten. Die erste und häufigste: dass ihnen nicht geglaubt wird. Weiter beklagen sie sich über fehlende Unterstützung, über die Hürden, welche ihnen entgegenstehen, dass sie ihre Situation x-mal zu berichten haben, über die unzureichenden rechtlichen Verfahren usw. Nicht zuletzt beklagen sie sich auch darüber, dass angeklagte Fachleute weiterhin ihren Beruf ausüben, obwohl sie fundamentalste Regeln ihrer Disziplin missachtet haben.

"How is it possible that professionals who are sworn to serve and protect the public, can sexually abuse their patients? That there is a single case of sexual abuse by a health care professional is wrong; that there are thousands is nearly impossible to believe, but that there are few prosecutions and no protection by the collegues is astounding." Dieses Zitat aus dem Bericht der «Task Force on the Sexual Abuse of Patients, Minister of Health, Ontario" [8] verdeutlicht prägnant die Problematik. Bezeichnenderweise finden diese Aspekte weder in der aktuellen «NeuenFehlerkultur-Diskussion" [9] noch in Qualitätssicherungsmassnahmen Berücksichtigung. In den Curricula werden sie ebenfalls ausgeblendet. Wahrscheinlich werden die epidemiologischen Zahlen von den Verantwortlichen massiv unterschätzt, obwohl Hinweise wie beispielsweise im «British Medical Journal» $[10,11]$ schon vor Jahren durch Inzidenzuntersuchungen die Situation in Europa belegten.

Für die Inzidenz in westlichen Industrienationen sind die neusten Zahlen aus Ontario [12] am aussagekräftigsten, welche auf einer Bevölkerungsbefragung beruhen, die im Rahmen einer regelmässig durchgeführten nationalen Datenerhebung 1999 unternommen wurde. Für den Zeitraum der zurückliegenden 5 Jahre gaben 1\% der Bevölkerung an, dass sie sexuelle Kontakte mit Fachleuten im Gesundheitswesen hatten. Auf die Schweiz übertragen wäre von jährlich 14000 betroffenen Personen auszugehen! Weitere $2 \%$ gaben für denselben Zeitraum an, sexuell gefärbte Bemerkungen und anzügliche Handlungen erlebt zu haben. Damit wurden bereits 1991 in Ontario erhobene Daten einer wesentlich kleineren Stichprobe bestätigt [13]. Die Fachleute in Kanada gehen von einer Dunkelziffer von rund 50\% in ihren Datenerhebungen aus, womit die effektiven Zahlen doppelt so hoch sein dürften. Selbstverständlich lassen sich derartige nationale Datenerhebungen nicht tel quel auf andere Länder übertragen. Die Zahlen sollen mangels verlässlicher Daten einzig einer Grössenabschätzung für unser Land dienen.

Die Arbeit der PABS dient der Wahrnehmung der Interessen von Patienten und ihren Angehörigen, die sich durch eine Ärztin oder einen Arzt unkorrekt behandelt fühlen. Damit schafft eine solche Stelle für eine Standesorganisation scheinbar ein Dilemma zum impliziten Auftrag der Wahrung der Interessen der einzelnen Mitglieder. Da sich jedoch ärztliches Handeln immer am Wohl und am Interesse des Patienten $\mathrm{zu}$ orientieren hat, löst sich dieses Dilemma auf. Es kann kaum im Interesse der Standesorganisation liegen, fehlbare Kollegen weiterhin zu schützen oder zu decken. Die PABS hilft mit, die Versorgungsqualität 
zu optimieren, da diejenigen Kolleginnen und Kollegen identifizierbar werden, die sich nicht an die anerkannten Regeln ärztlichen Handelns halten und solchermassen Patienten, wie auch dem ärztlichen Berufsstand als ganzes, schaden. Qualitätssicherung kann nicht nur "technische» Fragestellungen umfassen, sondern muss auch berufsethische Aspekte berücksichtigen.

\section{Vorgehensweise}

Die PABS ist gemäss einer «Politik der offenen Türe» konzipiert und soll ein niederschwelliges Beratungsangebot für Betroffene anbieten. In einem Reglement [1] ist die Arbeitsweise festgehalten. Für das Publikum wird eine Hotline eingerichtet mit einer speziell für die PABS reservierten Telefonnummer. Die initiale Beratung wird durch mindestens vier Ärztinnen und Ärzte gewährleistet. Sie werden durch die MedGes für ihre Aufgabe geschult. Die PABS hat keinerlei Fahndungsfunktion, sie hat auch keine sanktionierende Kompetenz. Andererseits muss klar festgehalten werden, dass jede Beratungsstelle nur so gute Arbeit leisten kann, wie sie ihr zugestanden wird. Es bedarf somit eines klaren Auftrages wie auch einer klaren Haltung seitens der Verantwortlichen innerhalb der MedGes, wie sie nun auch gegeben sind.

Um die beratenden Ärztinnen und Ärzte der PABS möglichst von Konflikten innerhalb der Ärzteschaft zu bewahren, wurde eine übergeordnete 3köpfige Steuerungsgruppe (SG-PAPS) eingesetzt, die den institutionalisierten Dialog mit anderen Instanzen sowohl innerhalb der Standesorganisation wie auch gegenüber öffentlichen Beratungsstellen und Behörden gewährleistet und als Schnittstelle zur PABS funktioniert. Die SG-PABS übernimmt gegenüber den beratenden Ärzten zudem eine Tutoriatsfunktion. Die SG-PABS formuliert zuhanden des Vorstandes standespolitische Anliegen, welche aus der Arbeit der PABS resultieren. Durch die vorbereitende Arbeitsgruppe wurde eine Guideline erarbeitet, welche die Arbeitsweise der PABS näher präzisiert. Die Guideline wurde durch den Vorstand geprüft und verabschiedet. In der Standesordnung FMH von 1996 zeigen sich verschiedene offene Fragen, die sich aus der Formulierung von Art. 4 ergeben: «Arzt und Ärztin dürfen ein sich aus der ärztlichen Tätigkeit ergebendes Abhängigkeitsverhältnis nicht missbrauchen, insbesondere darf das Verhältnis weder emotionell oder sexuell noch materiell ausgenützt werden.» [14] Ab welchem Zeitpunkt einer ärztlichen Tätigkeit gelten diese Bestimmungen? Wie lange sollen sie Gültigkeit haben - hört mit dem Behandlungsende die Verantwortlichkeit der Fachleute auf? Wie ist ein sexueller Missbrauch zu definieren, was versteht die Standesordnung unter: die sich ergebende Abhängigkeit? Die Steuerungsgruppe wird zuhanden der Mitglieder und des Publikums eine geeignete Publikationsform dieser Richtlinien erarbeiten, welche im Wartezimmer aufgelegt werden kann und in den Medien vorgestellt wird.

\section{Medizinethischer Diskurs}

Im wesentlichen bestimmen innerhalb der Medizinethik zwei deontologische Prinzipien das ärztliche Handeln [15]. Das erste verlangt: nihil nocere. Do not harm oder füge keinen Schaden zu. Das zweite fordert: facere bonum. Do the best oder helfe deinem Patienten nach bestem Wissen und Gewissen. Dass diese beiden Prinzipien durch sexuelle Missbräuche eklatant verletzt werden, ist evident und benötigt keine weitere Begründung.

In der medizinethischen Diskussion zur Thematik der sexuellen Missbräuche hat Cullen [16] fünf Argumente für eine «zero tolerance»-Haltung gegenüber sexuellen Handlungen angeführt. Das erste Argument beruht auf den empirischen Daten, welche belegen, dass sexuelle Handlungen im Rahmen ärztlicher Behandlungen in den meisten Fällen zu erheblichen Folgen führen und keineswegs als harmlose Begebenheiten abgetan werden können. Der Autor bezieht sich dabei auf das bereits erwähnte Editorial im «British Medical Journal» [6]. Gegen dieses Argument wird häufig von Ärzten selbst ins Feld geführt, dass gerade in ihrem Fall keine schlechten Folgen auftraten und diese These somit jeglicher Grundlage entbehre oder zumindest nicht verallgemeinert werden dürfe. Dem hält Cullen allerdings entgegen, dass sich keine prädiktiven Faktoren finden, welche eine Abschätzung erlauben, bei welchen Personen allenfalls gravierende Folgen auftreten können und bei welchen nicht. Er führt dazu aus: "Medicine has a history of charlatans who claim to be able to distinguish the special cases who will benefit from interventions that are, in general, dangerous. They claim skills not shared by their peers, and from which they make a personal gain.»

Wenn das vorstehende Argument angezweifelt wird, kann nur durch wissenschaftliche Studien belegt werden, dass sexuelle Handlungen meistens zu gravierenden Folgeschäden führen. Cullen ist entgegen der Meinung des Autors der Ansicht, dass die bisher vorliegenden Untersuchungen nicht geeignet sind, diese These zweifelsfrei zu belegen. Er ist der Ansicht, dass die schädigenden Auswirkungen bisher vorwiegend durch kasuistische Arbeiten belegt wurden, denen jedoch als Einzelfall keine allgemeinverbindliche Aussage zukommt. Cullen ist dennoch der Ansicht, dass das Argument der möglichen Folgeschäden in nachfolgenden Sinne Verwendung finden kann:

1. Sexuelle Kontakte zwischen Arzt und Patient sind manchmal mit gravierenden Folgen für den $\mathrm{Pa}-$ tienten verbunden.

2. Es gibt keine Patienten, deren Leben durch sexuelle Kontakte ihrer Ärzte im Rahmen von Behandlungen gerettet wurden.

3. Eine verlässliche Voraussage, welche Patienten gravierende Folgeschäden nach sexuellen Kontakten haben, ist nicht möglich.

4. Ärztliche Handlungen, die gelegentlich zu gravierenden Folgeschäden führen; Handlungen, welche zudem nie lebenserhaltend sind, und wo 
nie vorausgesagt werden kann, wer von derartigen Folgeschäden betroffenen sein kann, sollen nicht durchgeführt werden.

Conclusio: Sexuelle Kontakte zwischen Arzt und Patient sollten nie stattfinden. (Übersetzung W. Tschan)

Ein zweites Argument ist ein Prinzipienargument basierend auf den berufsethischen Anforderungen an einen Arzt. Allerdings gibt Cullen zu bedenken, dass die Grundsätze der Wahrung und Respektierung der Patientenautonomie gerade in der Diskussion um sexuelle Handlungen nicht a priori als Argument verwendet werden dürfen, da Ärzte häufig einen konsensuellen Kontakt zwischen zwei Erwachsenen geltend machen. Cullen geht jedoch nicht weiter auf die zugrundeliegenden Aspekte dieser Konsensualität ein. Hingegen hat Anton Leist in einem ausgezeichneten Aufsatz darauf hingewiesen, dass nicht jede konsensuelle Handlung auch ethisch vertretbar ist [17]. Häufig realisieren Betroffene erst viel später, dass sie manipuliert worden sind, oder dass sie einem "sanften" Druck nachgegeben haben, der nur unter einer Behandlungssituation denkbar ist. Es sei in diesem Zusammenhang auf das "slippery slope»-Konzept bei sexuellen Missbräuchen verwiesen, welches zunehmende Grenzüberschreitungen im Rahmen von ärztlichen Behandlungen postuliert, die jeweils isoliert betrachtet nicht unbedingt als schädigend zu bezeichnen sind - werden sie jedoch als ein zunehmender Vorgang verstanden, bis es schlussendlich zu Grenzverletzungen und sexuellen Handlungen kommt, wird auch das planende und zielgerichtete Vorgehen von Fachleuten erkennbar. Unter Behandlungsbedingungen erfährt die objektive Einschätzung auf seiten der Patienten eine Einschränkung, indem der einzelne sich weitgehend dem Urteil der Fachperson überlässt.

Das dritte Argument ist ein tugendethisches und definiert den "guten" Arzt. Diese tugendethischen Anforderungen fliessen beispielsweise in die ärztliche Ausbildung ein. Die Tugenden basieren auf den berufsethischen Anforderungen und sind in der ärztlichen Standesordnung formuliert. Dem steht einzig entgegen, dass Fälle denkbar sind, wo ohne Verletzung dieser tugendethischen Ansätze ein Arzt oder ein Patient sich gegenseitig ineinander verlieben.

Ein viertes Argument ist ein A-priori-Argument. Dieses Argument lässt sich wie folgt charakterisieren:

1. Die Arzt-Patienten-Beziehung hat die und die Eigenschaften.

2. Eine sexuelle Beziehung zwischen zwei Menschen kann genau diese Eigenschaft nicht haben.

Conclusio: Patienten sind keine Sexualpartner.

Dieses Argument beruht auf der mehr intuitiven Haltung vieler Ärzte, dass sie ihre eigenen Angehörigen nicht behandeln sollen. $\mathrm{Zu}$ den ärztlichen Eigenschaften gehört beispielsweise die Notwendigkeit fachlicher und emotionaler Distanz. Umgekehrt gelten solche Werte sicher nicht als Voraussetzung für eine glückliche intime Beziehung. Letztendlich blei- ben jedoch solche Überlegungen weitgehend Ansichtssache, auch wenn sie von vielen geteilt werden. Als letztes und stärkstes Argument führt Cullen ein kontrafaktisches Argument an:

Wenn sexuelle Kontakte zwischen Ärzten und Patienten gestattet wären, würde dies zu inakzeptablen Konsequenzen führen.

Conclusio: Sexuelle Kontakte zwischen Ärzten und Patienten sind nie erlaubt.

Als mögliche inakzeptable Konsequenzen führt Cullen den resultierenden Vertrauensverlust in die ärztliche Kunst an. Ferner könnten durch solcherart handelnde Ärzte die Aufrechterhaltung fachlicher und emotionaler Grenzen nicht mehr gewährleistet werden. Nach Cullen ist es somit moralisch verwerflich, wenn Ärzte mit ihren Patienten einen sexuellen Kontakt eingehen. Das letzte und kontrafaktische Argument gilt dabei für Cullen als praktisch nicht zu widerlegende These. Der Autor leitet daraus die Forderung ab, dass nur eine "zero tolerance»-Antwort gegenüber sexuellen Kontakten im Rahmen von ärztlichen Beziehungen angemessen sein kann.

Es scheint wesentlich, der Frage nachzugehen, welche grundlegende Bedeutung das Vertrauensverhältnis zu den Fachleuten im Gesundheitswesen eigentlich hat. Den Ärzten werden Eingriffe am Körper erlaubt und zugestanden, die nicht einmal den nächsten Angehörigen zugestanden werden. Sich einer Operation zu unterziehen, eine Narkose zu tolerieren, ja schon nur eine Injektion zuzulassen, ist nur denkbar und möglich, wenn den ausführenden Fachleuten Vertrauen entgegengebracht wird, dass sie erstens ihr Handwerk verstehen und zweitens für den jeweiligen Patienten das bestmögliche tun. In Analogie gelten diese Ausführungen auch für seelischen Aspekte, wo erst das Vertrauen einer Person ermöglicht, dass sie sich öffnet und belastende Dinge auch mitteilen kann. Es ist unbestritten, dass dies letztendlich erst den Heilungsprozess ermöglicht [18]. Die Diskussionen um Abtreibung und Sterbehilfe weisen unter anderem auf die symbolischen Aspekte hin, welche mit der Bedeutung der Arzt-Patienten-Beziehung verbunden sind und welche mit zutiefst existentiellen Ängsten erklärt werden können. Das Gefühl des Ausgeliefertseins auf Leben und Tod gegenüber den ärztlichen Fachpersonen erfordert eine sehr sorgfältige Abwägung solcher Entscheide. Nicht ganz zu Unrecht wird in diesen Diskussionen immer wieder die unrühmliche Mitbeteiligung der Schulmedizin am Blutterror der Nationalsozialisten in Deutschland als Mahnmahl angeführt [19]. Umgekehrt hat erst das Erkennen der erschreckenden Zusammenhänge zu einem verstärkten medizinethischen Diskurs beigetragen [20]. Mit der Verabschiedung des Nürnberger Codex wurde innerhalb der Medizin ein Weg gesucht, sich im Rahmen ärztlicher Behandlungen die Zustimmung des Patienten zu sichern [21]. Der "Informed Consent" wurde in der Folge als Standard ärztlichen Handelns formuliert. Die Medizin ist geradezu darauf angewiesen, dass diejenigen, welche ihre Dienste in Anspruch nehmen, 
das auch vertrauensvoll tun können. Die Berufsanforderungen sowie die Berufszulassung sind deswegen zu Recht stark reglementiert und unterliegen der gesellschaftlichen und staatlichen Kontrolle.

\section{Ausblick}

Die Beratungstätigkeit der PABS wird wissenschaftlich begleitend evaluiert werden. Die bisherigen Beratungskonzeptionen beruhen praktisch ausschliesslich auf den theoretisch und praktisch fundierten Annahmen der Fachleute, ohne dass die Bedürfnisse und Erwartungen der betroffenen Opfer systematisch berücksichtigt wurden. Bisher wurde nach Wissen des Autors einzig im strafrechtlichen Bereich eine Überprüfung der Opferberatung durchgeführt [22]. Die Ergebnisse der Evaluation sollen im Sinne eines Feedbackmechanismus in die Grundkonzeption der PABS einfliessen. Es ist vorgesehen, auch die Langzeiteffekte zu validieren.

Weiter wird die MedGes eine Arbeitsgruppe einsetzen, welche den Umgang mit Tätern bzw. angeschuldigten Kolleginnen und Kollegen reflektieren soll. Die bisher in unserem Lande ergriffenen Massnahmen haben sich im internationalen Vergleich als reichlich blauäugig und ungenügend erwiesen. So wurde beispielsweise schon vor Jahren gefordert, die Curricula der Humanmedizin entsprechend anzupassen. Weltweit zeigt sich die Tendenz, durch ein zumindest temporäres Berufsverbot den Patienten einen Schutz vor weiteren Missbräuchen zu gewährleisten [23]. Nur durch eine gezielte Untersuchung und Beurteilung der missbrauchenden Fachleute sowie Entwicklung geeigneter Behandlungs- und Präventivstrategien lassen sich die anstehenden Probleme und Fragestellungen adäquat angehen. Mittels eines etablierten Assessmentverfahrens und eines sich daran anschliessenden Rehabilitationsprogrammes im Sinne eines "boundary trainings" [24] kann die Berufszulassung wieder erreicht werden. Allenfalls muss durch ein Monitoring die weitere Berufstätigkeit periodisch überprüft werden [25]. Die Bezeichnung "Täter» mag für viele Kolleginnen und Kollegen befremdend klingen. Darin zeigt sich jedoch gerade unsere heutige Optik auf. Da wir in unserem Land im Gegensatz zu vielen anderen Staaten keine eindeutigen strafgesetzlichen Bestimmungen zum Patientenschutz kennen, werden sexuelle Missbräuche auch nicht als Straftatbestände wahrgenommen. Nach wie vor besteht die verbreitete Meinung eines «Kavaliersdeliktes", wenn es innerhalb fachlicher Beziehungen zu sexuellen Kontakten kommt. Denjenigen, welche diese Ansicht teilen, sei das Studium des entsprechenden Bundesgerichtsurteils von 1998 [26] empfohlen. Weiter sei mittels des nachfolgenden Zitates auf die persönliche Situation von Fachleuten verwiesen: "Unfortunately [...] there has been practically no discussion of how the power relationship can become topsy-turvy when the client decides to file a complaint" [27]. Deswegen sind die Curricula entsprechend anzupassen, damit bereits auf Ausbildungsstufe die jungen Kolleginnen und Kollegen die nöti- gen Kenntnisse vermittelt bekommen [28-30]. Das gleiche gilt für die postgraduierte Weiterbildung [31, 32].

Die administrativen Behörden wie auch die öffentlichen Gerichte müssen dafür gewonnen werden, den durch die Ärzteschaft formulierten Grundsätzen der Standesordnung Nachachtung zu verschaffen. Es nutzt nichts, betroffenen Opfern gute Ratschläge zu erteilen, wenn ihre Anliegen keinerlei Aussicht auf Erfolg haben. In der Schweiz hat die Ärzteschaft keinerlei sanktionierende Kompetenz. Durch Austritt aus der kantonalen Ärzteschaft kann man sich elegant jeder ehrenrätlichen Auflage entziehen. Im Sinne der staatlichen Gewaltenteilung sollte die Ärzteschaft diese Kompetenz auch nicht anstreben, sondern vielmehr durch Formulierung eigener berufsethischer Grundsätze den "State of the Art» festlegen und die administrative und richterliche Beurteilung den dazu vorgesehenen Instanzen überlassen.

Weltweit wurde erkannt, dass Mitarbeiterinnen und Mitarbeiter von Opferberatungsstellen selbst nicht davor gefeit sind, ihrerseits Missbräuche zu verüben. Beispielsweise fand sich in der NZZ vor kurzem eine entsprechende Agenturmeldung über eine Kinderschutzstelle [33]. Ebenso sind entsprechende Beispiele aus der Literatur bekannt [34]. Es müssen daher durch die Verantwortlichen die notwendigen Vorkehrungen getroffen werden. Der Autor ist in Zusammenarbeit mit Audit- und Zertifizierungsunternehmen an der Ausgestaltung entsprechender Verfahren beteiligt. Durch Implementierung solcher Massnahmen geben die ärztlichen Standesorganisationen eine "Good Working Practice" vor. Sobald konkrete Vorgehensweisen erarbeitet sind, wird die SG-PABS zusammen mit dem Vorstand MedGes die Implementierung eines derartigen Controllinginstrumentes zu prüfen haben.

Weiter ist vorgesehen, die umliegenden Kantone zur Partizipation einzuladen. In der Nordwestschweiz stossen auf engem Raum fünf Kantone aneinander. Gerade im ambulanten Bereich zeigt sich, dass die kantonale Organisation des Gesundheitswesens eine Fiktion ist, die der Praxisrealität in keiner Weise entspricht. Durch eine geographische Ausweitung des Tätigkeitsbereiches der PABS kann zudem der Befangenheitssituation der beratenden Ärztinnen und Ärzte besser begegnet werden. Und nicht zuletzt versprechen überkantonale Lösungen auch bessere Ressourcennutzungen an geeigneten Fachleuten, die über entsprechende Kenntnisse verfügen und bereit sind, solche Aufgaben zu übernehmen.

Schliesslich wird auch die Standesordnung FMH überarbeitet und angepasst werden müssen. Wir möchten alle einladen, sich an diesem spannenden Dialog über die Ausgestaltung des Arztberufes im 21. Jahrhundert aktiv zu beteiligen. Durch eine proaktive Auseinandersetzung mit solchen Themen können wir eine reaktive und defensive Vorgehensweise verhindern. Es wird unserem Ansehen nicht schaden, wenn wir schwierige Themen aufgreifen und die Öffentlichkeit erkennen kann, dass sowohl Qualitätssicherung in der ärztlichen Arbeit wie auch Patientensicherheit nicht nur leere Worthülsen sind. 


\section{Literatur}

1 Reglement der Patienten-Anlauf- und -Beratungsstelle Basel (PABS). Medizinische Gesellschaft Basel, 29. März 2001.

2 Gesetz betreffend Ausübung der Berufe der Medizinalpersonen und der Komplementärmedizin. Vom 26. Mai 1879. Kanton Basel-Stadt.

3 Herman J. Trauma and Recovery. New York: Basic Books; 1992.

4 Van der Kolk BA, McFarlane AC, Weisaeth L. Traumatic Stress - Grundlagen und Behandlungsansätze. Paderborn: Junfermann Verlag; 2000.

5 Fischer G, Riedesser P. Lehrbuch der Psychotraumatologie. München: E. Reinhardt Verlag; 1999.

6 Foa EB, Keane TM, Friedman MJ. Effective Treatments for PTSD. Practice Guidelines from the International Society for Traumatic Stress Studies. New York; The Guilford Press; 2000.

7 Kinderschutz - Adressverzeichnis von Hilfs- und Beratungsstellen in der Schweiz im Zusammenhang mit Kindesmisshandlungen. Zentralstelle für Familienfragen, Bundesamt für Sozialversicherungen, Bern, EDMZ 318.809 d.

8 Special Task Force on the Sexual Abuse of Patients, Minister of Health and Long-Term Care and Health Professions Regulatory Advisory Council. Executive Summary of the Final Report. Toronto, Canada. June 20, 2001.

9 FMH-Tagung «Risiken, Fehler und Patientensicherheit bei den medizinischen Behandlungen». Inselspital, Bern, 25. Januar 2001.

10 Fahy T, Fisher N. Sexual contact between doctors and patients. Br Med J 1992;304:1519-20.

11 Wilbers D, Veenstra G, van de Wiel HBM, Weijmar Schultz WCM. Sexual contact in the doctor-patient relationship in the Netherlands. Br Med J 1992;304:1531-4.

12 Manzer J. Conduct unbecoming. Med Post 2000;36(17):1.

13 Task Force on Sexual Abuse of Patients. The Final Report. Toronto: College of Physicians and Surgeons of Ontario; 1991.

14 Standesordnung FMH vom 12. Dezember 1996. Schweiz Ärztezeitung 1997;78(11):373-83.

15 Sass HM. Medizinethik. In: Pieper A, Thurnherr U. Angewandte Ethik. Eine Einführung. München: Verlag C. H. Beck; 1998. S. 80-109.

16 Cullen RM. Arguments for zero tolerance of sexual contact between doctors and patients. J Med Ethics 1999;25:482-6.

17 Leist A. Ethik ohne Grundlagen. Referat an der Ethiktagung in Brixen, 25. September 2000.

18 Pennebaker JW. Emotion, Disclosure and Health. Washington, DC: American Psychological Association; 1995.
19 Kater MH. Doctors under Hitler. Chapel Hill: University of North Carolina Press; 1989. Deutsch: Ärzte als Hitlers Helfer. Hamburg: Europa Verlag; 2000.

20 Schöne-Seifert B. Medizinethik. In: Nida-Rümelin J. Angewandte Ethik. Die Bereichsethiken und ihre theoretische Fundierung. Stuttgart: A. Kröner Verlag; 1996. S. 552-648.

21 Tröhler U. Das ärztliche Ethos und die Kodifizierung von Ethik in der Medizin. In: Bondolfi A, Müller HJ. Medizinische Ethik im ärztlichen Alltag. Basel: EMH Schweizerischer Ärzteverlag; 1999. S. 39-61.

22 Baurmann MC, Schädler W. Das Opfer nach der Straftat seine Erwartungen und Perspektiven. Red. korr. Nachdruck. Wiesbaden: Bundeskriminalamt; 1999.

23 Hatch D. General Medical Council programmes for reduction of errors and protection of patients. Schweiz Ärztezeitung 2001;82(25):1344-7.

24 Tschan W. Missbrauchtes Vertrauen - Grenzverletzungen in professionellen Beziehungen. Basel: Karger Verlag; 2001.

25 Physician Diversion Program. Sacramento: Medical Board of California; 2000.

26 Bundesgerichtsentscheid 12.01.1998 (BGE 124 IV 13).

27 Luepker ET, Schoener GR. Sexual Involvement and the Abuse of Power in Psychotherapeutic Relationships. In: Schoener G, Milgrom JH, Gonsiorek JC, Luepker ET, Conroe RM. Psychotherapists' sexual involvement with clients: Intervention and Prevention. Minneapolis: Walk-In Counseling Center; 1989. p. 65-72.

28 Robinson GE, Stewart DE. A curriculum on physician-patient sexual misconduct and teacher-learner mistreatment. Part 1: content. Can Med Assoc J 1996;154(5):643-9.

29 Robinson GE, Stewart DE. A curriculum on physician-patient sexual misconduct and teacher-learner mistreatment. Part 2: teaching method. Can Med Assoc J 1996;154(7):1021-5.

30 Gorton GE, Samuel SE. A national survey of training directors about education for prevention of psychiatrist-patient sexual exploitation. Academic Psychiatry 1996;20,2:92-8.

31 Gabbard Glen 0. Lessons to be learned from the study of sexual boundary violations. Am J Psychother 1996;50(3): 311-22.

32 Gutheil TG, Gabbard G0. The concept of boundaries in clinical practice: theoretical and risk-management dimensions. Am J Psychiatry 1993;150(2):188-96.

33 Kinderschützer in Luxemburg als Kinderschänder. dpa-Meldung in: Neue Zürcher Zeitung Nr. 155, 7./8. Juli 2001.

34 Simon RI. Bad men do what good men dream. Washington, DC: American Psychiatric Press; 1999. 